\title{
ANALYSIS OF PARAMETERIZATION VALUE REDUCTION OF SOFT SETS AND ITS ALGORITHM
}

\author{
Mohammed Adam Taheir Mohammed ${ }^{1 *}$, Wan Maseri Wan Mohd ${ }^{1}$, Ruzaini \\ Abdullah Arshah ${ }^{1}$, M. Mungad ${ }^{2}$, Edi Sutoyo ${ }^{2}$ and Haruna Chiroma ${ }^{3,4}$ \\ ${ }^{1}$ Faculty of Computer Systems \& Software Engineering, Universiti Malaysia Pahang, \\ 26300 Gambang, Pahang, Malaysia \\ ${ }^{2}$ Department of Information System, \\ ${ }^{3}$ Department of Artificial Intelligence, University of Malaya \\ 50603 Pantai Valley, Kuala Lumpur, Malaysia \\ ${ }^{4}$ Computer Science Department, Federal College of Education (Technical), Gombe, \\ Nigeria \\ *Email: mohammed_taher30@yahoo.com \\ Phone: +601126031065
}

\begin{abstract}
In this paper, the parameterization value reduction of soft sets and its algorithm in decision making are studied and described. It is based on parameterization reduction of soft sets. The purpose of this study is to investigate the inherited disadvantages of parameterization reduction of soft sets and its algorithm. The algorithms presented in this study attempt to reduce the value of least parameters from soft set. Through the analysis, two techniques have been described. Through this study, it is found that parameterization reduction of soft sets and its algorithm has yielded a different and inconsistency in suboptimal result.
\end{abstract}

Keywords: Microwave Irradiation, Nanohexagons, Nanomaterials, Photoluminescence, Quantum Size Effect, Semiconductors.

\section{INTRODUCTION}

Nowadays, there are major issues in redundancy of data on an ongoing basis as a result of development, especially in decision-making; therefore the need of extra memory is necessary which affects the storage and copies space, thus, it is necessary to reduce the amount of data. For this reason, researchers should develop smart techniques to address this problem by optimal solutions as well as taken into account sub optimal, so these intelligent applications must inherit the characteristic of original soft data. Uncertain data occurs on many real life problems in engineering, medical, social and medical sciences (Maji and Biswas, 2002) which need to be managed and solved. Soft set theory is one of mathematical principles that can be used to solved uncertain data problems (Molodtsov, 1999). The theory of soft set was introduced by Molodtsov (1999) as a new way for handling uncertain data. Molodtsov (1999) figured out that "one of the main soft set theory advantages that it is free from the inadequacy of the parameterization tools, unlike in the theories of fuzzy set, probability and interval mathematics".

The result of (Chen et al., 2005) on the parameterization reduction of soft sets and its algorithm will be analyzed to finds its correctness, further the result by (Ma et al., 2011) will be discussed in terms of whether it has achieve the optimal and sub optimal decisions. This paper is organized as follows: Section 2 describes the concept of 
soft set theory and section 3 describes materials and methods used in previous works. Section 4 discusses the result of two previous reduction work and end up with conclusions.

\section{SOFT SET THEORY}

In this section, we highlight some of the ideas of soft set theory. To avoid difficulties an adequate parameterization must be used. Let $U$ be a universe set which have parameters in relation to object in the universe. The set denote the power set of. The definition of soft set is given as follows

Definition (See (Pawlak and Skowron, 2007)) in data set A pair is denoted as a soft set over where the function is a mapping the relation given by. In other words, the parameterized family subset is belong to U. Every set, from this family may be considered as the set of $\varepsilon$-elements of the soft set, or as the set of approximate elements of the soft set. As an illustration, let us consider the following example.

Example 2.1. Let a soft set, study the communication prowess representation among selected university students (Rose et al., 2011).

Let assume that there are eighteen students using different six commendations programs such as email, facebook, blog, friendsters, Yahoo messenger and sms. While the commendations programs usage are variant from student to another, namely: the university students and are the six communications programs. It maps given by student who preferable such communication prowess, where is to be filled in by one of the parameters. For example represent the student who preferable facebook as, while shows the student who preferable friendster and their value represented by as shown in Table 1.

Table 1. Tabular representation of a soft set

\begin{tabular}{llllllll}
\hline$U / P$ & $p_{1}$ & $p_{2}$ & $p_{3}$ & $p_{4}$ & $p_{5}$ & $p_{6}$ & $f()$. \\
\hline$u_{1}$ & 1 & 0 & 1 & 0 & 0 & 0 & 2 \\
$u_{2}$ & 1 & 1 & 1 & 1 & 1 & 1 & 6 \\
$u_{3}$ & 1 & 1 & 1 & 1 & 1 & 1 & 6 \\
$u_{4}$ & 1 & 1 & 1 & 1 & 1 & 1 & 6 \\
$u_{5}$ & 0 & 1 & 0 & 0 & 1 & 0 & 2 \\
$u_{6}$ & 0 & 0 & 1 & 0 & 1 & 0 & 2 \\
$u_{7}$ & 0 & 0 & 0 & 0 & 1 & 0 & 1 \\
$u_{8}$ & 1 & 1 & 1 & 1 & 1 & 1 & 6 \\
$u_{9}$ & 1 & 1 & 1 & 1 & 1 & 1 & 6 \\
$u_{10}$ & 1 & 1 & 1 & 1 & 1 & 1 & 6 \\
$u_{11}$ & 1 & 0 & 1 & 0 & 1 & 0 & 3 \\
$u_{12}$ & 0 & 1 & 0 & 1 & 0 & 0 & 2 \\
$u_{13}$ & 1 & 1 & 1 & 1 & 1 & 1 & 6 \\
$u_{14}$ & 1 & 1 & 0 & 0 & 0 & 0 & 2 \\
$u_{15}$ & 1 & 1 & 1 & 1 & 1 & 1 & 6 \\
$u_{16}$ & 1 & 1 & 1 & 1 & 1 & 1 & 6 \\
$u_{17}$ & 1 & 1 & 1 & 1 & 1 & 1 & 6 \\
$u_{18}$ & 0 & 0 & 1 & 1 & 1 & 0 & 3 \\
\hline
\end{tabular}


In this section we analyzed two previous reduction algorithms as follows. Analysis of attribute reduction of soft set in (Maji et al., 2002). The idea of soft set operations was firstly proposed by Maji et al. (2002). In (Maji et al., 2002), the application of soft set theory to a decision making problem with the help of Pawlak's rough mathematics is presented. Maji et al. (2002) chose the decision from objects based on maximal weighted value. The two major drawbacks of Maji's proposal is that sub optimal decision yield different weighted value from the identified reduction of soft sets and inconsistencies. Therefore, inconsistencies increase the cost of choices in decision making. Consider Mr. X wants to purchase construction's items and the question why we let Mr. X pay more even involve him in risk off course because of uncertainly. According to Maji et al. (2002), the partition based on maximum value of choices based in Table 1 is given as follows,

$$
U / E=\left\{\begin{array}{l}
\left\{u_{1}\right\},\left\{u_{2}, u_{3}, u_{4}, u_{8}, u_{9}, u_{10}, u_{13}, u_{15}, u_{16}, u_{17}\right\} \\
\left\{u_{5}\right\},\left\{u_{6}\right\},\left\{u_{7}\right\},\left\{u_{11}\right\},\left\{u_{12}\right\},\left\{u_{14}\right\},\left\{u_{18}\right\}
\end{array}\right\}
$$

Therefore, the first sub-optimal decision of objects are $u_{11}$ and $u_{18}$ and the second suboptimal decision of objects are $u_{1}, u_{5}, u_{6}, u_{12}, u_{14}$. The last choice is $u_{7}$. From [1], let $R \subset E$, where $D=\left\{p_{1}, p_{2}, p_{4}, p_{5}\right\}$, is still produces the same partition of optimal decision of $E$ that equal to partition $U / E=U / D$ ( Mohammed et al., 2014).

From optimal decision that $U / D$ is an invariant to $U / E$ and therefore can be considered as an (rough) attribute reduced of $E$ still $\left\{u_{2}, u_{3}, u_{4}, u_{8}, u_{9}, u_{10}, u_{13}, u_{15}, u_{16}, u_{17}\right\}$, denoted by the maximum value is 4 . From such partition, the optimal decision of objects are $u_{2}, u_{3}, u_{4}, u_{8}, u_{9}, u_{10}, u_{13}, u_{15}, u_{16}$ and $u_{17}$. The reduction of Table 1 based on [3] as shown in Table 2.

$$
U / D=\left\{\begin{array}{l}
\left\{u_{1}\right\},\left\{u_{2}, u_{3}, u_{4}, u_{8}, u_{9}, u_{10}, u_{13}, u_{15}, u_{16}, u_{17}\right\},\left\{u_{5}\right\} \\
\left\{u_{6}\right\},\left\{u_{7}\right\},\left\{u_{11}\right\},\left\{u_{12}\right\},\left\{u_{14}\right\},\left\{u_{18}\right\}
\end{array}\right\}
$$

Table 2. Reduction analysis

\begin{tabular}{llllll}
\hline$U / P$ & $p_{1}$ & $p_{2}$ & $p_{4}$ & $p_{5}$ & $f()$. \\
\hline$u_{1}$ & 1 & 0 & 0 & 0 & 1 \\
$u_{2}$ & 1 & 1 & 1 & 1 & 4 \\
$u_{3}$ & 1 & 1 & 1 & 1 & 4 \\
$u_{4}$ & 1 & 1 & 1 & 1 & 4 \\
$u_{5}$ & 0 & 1 & 0 & 1 & 2 \\
$u_{6}$ & 0 & 0 & 0 & 1 & 1 \\
$u_{7}$ & 0 & 0 & 0 & 1 & 1 \\
$u_{8}$ & 1 & 1 & 1 & 1 & 4 \\
$u_{9}$ & 1 & 1 & 1 & 1 & 4 \\
$u_{10}$ & 1 & 1 & 1 & 1 & 4 \\
$u_{11}$ & 1 & 0 & 0 & 1 & 2 \\
$u_{12}$ & 0 & 1 & 1 & 0 & 2 \\
$u_{13}$ & 1 & 1 & 1 & 1 & 4 \\
$u_{14}$ & 1 & 1 & 0 & 0 & 2 \\
$u_{15}$ & 1 & 1 & 1 & 1 & 4
\end{tabular}




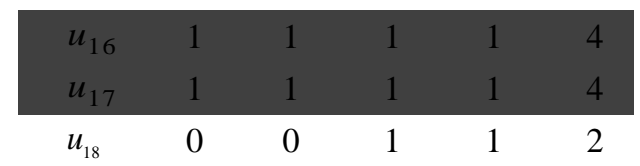

Optimal decision of object is one of the objects in the set, which is not same as that suboptimal decision derived from E, i.e. And then, the second sub-optimal decision of object is now objects in instead of since it was prior the process of reduction. Finally the reduction result is $66 \%$, but still there is inconsistency for parameterization reduction, Chen et al. (2005), has defined where the term of final decision.

\section{Analysis of parameterization reduction of soft set in (Chen et al., 2005)}

The reduction algorithm introduced by Chen et al. (2005), highlight their main objective is that to solve the inconsistency that made by Maji et al. Chen also reduce the inconsistency from Maji et al.'s algorithm successfully. Chen highlighted that suppose the list of parameters which induce maximum choice portions is Q and independent, to narrow the inconsistency from $Q$ to any parameter if deleted, induce same maximum choice partition, implies the reduction from Q. For parameterization reduction, Chen et al. (2005), has defined $f_{E}\left(h_{i}\right)=\sum_{j} h_{i j}$ where $h_{i j}$ are the entries in the Table representing $(F, E)$ and defined $M_{E}$ as group of objects in $\mathrm{U}$ which has the maximum value of choices $f_{E}$.

The reduction of $\mathrm{A}$ is determine if $\mathrm{A}$ is indispensable and $M_{A}=M_{E}$. As in Table 1, the set of parameters $A=\left\{p_{1}, p_{2}, p_{3}\right\}$ have

$$
M_{E-A}=\left\{\begin{array}{l}
u_{2}, u_{3}, u_{4}, u_{8}, u_{9}, u_{10}, u_{13}, \\
u_{15}, u_{16}, u_{17}
\end{array}\right\}
$$

Therefore it indispensable and $M_{E-A}=M_{E}$, so the set $A=\left\{p_{1}, p_{2}, p_{5}\right\}$ is reduction of $\mathrm{E}$ as shown in Table 3.

As shown in the Table 3, Chen et al. has successfully provide consistency in the optimal decision of objects but failed in providing consistency for objects sub optimal decision.

The first sub-optimal decision of object is , which is different from original first sub-optimal decision of object derived from $\mathrm{E}$, which is in the set of .

The major disadvantage is again inconsistency as shown in the selection of object suboptimal decision. 
Table 3. A binary table of representation of A based on

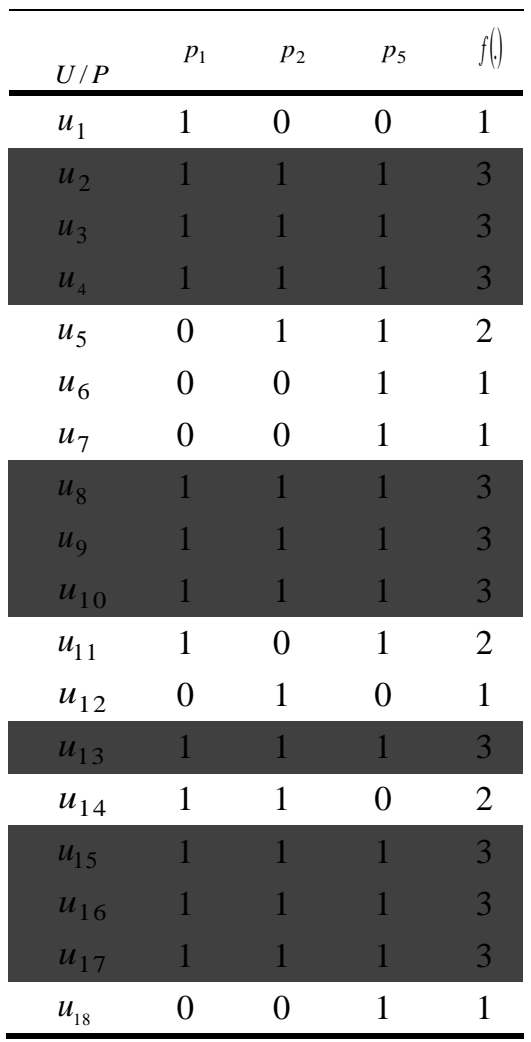

Also, the most glaring issue here is inconsistency as shown in the selection of the next sub-optimal decision of objects. The last sub-optimal decision of objects has now become $\{\mathrm{u} 1, \mathrm{u} 6, \mathrm{u} 7, \mathrm{u} 12, \mathrm{u} 18\}$ instead of as it was prior to the process of reduction. Basically, in this case, the data size has been shrunk up to $50 \%$, but still a cost of the expense of inconsistency.

\section{Analysis of Parameterization Value Reduction of Soft Set in [4]}

In the year 2011, Ma et al. (2011) introduced a technique to generate result based on the idea of Chen et al. (2005) which can be achieved by heuristic algorithm since it is able to identify each object in optimal choice and delete the remaining values.

Their algorithm as follows:

1-Accept soft set as input.

2-Delete parameter value denoted by 0 .

3-For every object in maximum choices partition, keep one value of 1 in their corresponding objects.

After classifications of original choices in Table 4 therefore the reduction of Table 1 based on (Ma et al., 2011), is same as the reduction in Table 3. 


\section{RESULT AND DISCUSSION}

Based on Table 1, the result of Maji et al., (2002) was incorrect since it focus only on optimal decision while sub optimal decisions not satisfied the characteristics of parameter reduction, therefore their sub optimal result varies to original result.

Based on Table 1, the result of Chen et al., (2005) reduce the inconsistency from Maji et al., (2002) but still shared the problem of sub optimal results in (Maji et al., 2002) by incorrect result. In (Ma et al., 2002) it reduce the least parameters which makes the optimal choice not change. The disadvantage of sub optimal decision results in (Ma et al., 2002).

Table 4.Parameterization Value Reduction of Soft Set based on Table 1

\begin{tabular}{cccccccc}
\hline$U / P$ & $p_{1}$ & $p_{2}$ & $p_{3}$ & $p_{4}$ & $p_{5}$ & $p_{6}$ & $f()$. \\
\hline$u_{1}$ & - & - & - & - & - & - & 0 \\
$u_{2}$ & - & - & - & - & - & 1 & 1 \\
$u_{3}$ & - & - & - & - & 1 & - & 1 \\
$u_{4}$ & - & - & - & 1 & - & - & 1 \\
$u_{5}$ & - & - & - & - & - & - & 0 \\
$u_{6}$ & - & - & - & - & - & - & 0 \\
$u_{7}$ & - & - & - & - & - & - & 0 \\
$u_{8}$ & - & - & 1 & - & - & - & 1 \\
$u_{9}$ & - & 1 & - & - & - & - & 1 \\
$u_{10}$ & 1 & - & - & - & - & - & 1 \\
$u_{11}$ & - & - & - & - & - & - & 0 \\
$u_{12}$ & - & - & - & - & - & - & 0 \\
$u_{13}$ & - & - & - & - & - & 1 & 1 \\
$u_{14}$ & - & - & - & - & - & - & 0 \\
$u_{15}$ & - & - & - & - & 1 & - & 1 \\
$u_{16}$ & - & - & - & 1 & - & - & 1 \\
$u_{17}$ & - & - & 1 & - & - & - & 1 \\
$u_{18}$ & - & - & - & - & - & - & 0 \\
\hline
\end{tabular}

\section{CONCLUSION}

It has been shown from our analysis that reduction from (Maji et al., 2002) is only successful at maintaining the maximum choice, but is not totally correct due to variant in sup optimal decisions. Also in (Chen et al., 2005), it has successfully maintained inconsistency from (Maji et al., 2002) but not provide consistency in sub optimal decisions which has sub optimal problem. While the result of (Ma et al., 2002), has disadvantage of having sub optimal decision. 


\section{REFERENCES}

Chen. D., Tsang, E.C.C, Yeung, D.S. and Wang, X. (2005). The Parameterization Reduction of Soft Sets and its Applications. Computers and Mathematics with Applications, 49, 757-763.

Ma, X., Sulaiman, N., Qin, H. (2011). The Parameterization Value Reduction of Soft Sets and its Applications. IEEE CHUSER, Penang.

Maji P.K, Roy A.R and Biswas, R. (2002). An applicationof soft sets in a decision making problem. Computer and Mathematics with Application, 44, 1077-1083.

Mohammed, M.A.T., Mohd, W.M.B.W., Arshah, R.B.A. and Yao, L. (2014). Parameter Reduction Comparisons. Asian Academic Research Associates AARJSH, 19 (1).

Molodtsov D. (1999). Soft set theory-first results. Computers and Mathematics with Applications, 37, 19-31.

Pawlak. Z. and Skowron, A. (2007). Rudiments of roughsets. Information Sciences, 177 (1), 03-27.

Rose, A.N.M., Awang, M.I., Hassan, H., Hassan, A.H., Herawan, T. and Deris. M.M. (2011). Hybrid Reduction in Soft Set Decision Making. Springer-Verlag Berlin Heidelberg ICIC 2011. LNCS 6838, 108-115. 